\title{
Isometric-Circle Interpretation of Bilinear Transfor- mation and its Application to VSWR Minimization ${ }^{1}$
}

\author{
James G. Rudolph and David K. Cheng \\ Electrical Engineering Department, Syracuse University, Syracuse, N.Y.
}

(Received Dec. 16, 1964; revised Mar. 23, 1965)

\begin{abstract}
A technique using an isometric-circle interpretation of a bilinear transformation has been developed for minimizing the input VSWR of a transmission line consisting of multiple discontinuities joined by line sections. It makes possible the determination not only of the minimum attainable input VSWR but also of the required line lengths between the successive discontinuities. The case for $N$ real and equal discontinuities is particularly simple, and the necessary conditions for obtaining a perfect match are established. Numerical examples are given.
\end{abstract}

\section{Introduction}

The use of a long transmission line (or waveguide) for transporting signal power from a source to a distant load is a common practice in communication engineering. A typical example can be found in the transmission circuit between a transmitter and its associated radiating antenna. In such cases, even if the external circuitry is matched to the connecting line, reflections may arise from discontinuities in the line itself due to the existence of rotary joints which enable the antenna to scan and of connectors which join sections of line together. Input VSWR minimization for a transmission line with multiple discontinuities presents itself as an important systems problem.

By using appropriate models for both the discontinuities and the line, an analytical expression for the input VSWR or the corresponding reflection coefficient can be derived in a straightforward manner. However, the resulting expression is difficult to interpret because it depends in a complicated way upon the properties of the discontinuities as well as upon the spacings between them. The fact that partial minimization achieved by moving one discontinuity at a time does not represent an overall optimum solution further complicates the situation.

This paper attacks the problem by making use of an isometric-circle method for interpreting a bilinear transformation. When a transmission line consists of multiple discontinuities joined by line sections, a bilinear transform relationship can be established between the reflection coefficients at successive discontinuities. The technique to be presented in the following sections enables one to determine not only the minimum attainable input VSWR under a given situation but also the required line lengths joining the discontinuities.

\section{General Formulation for Equal Discontinuities}

Consider a long and perfectly terminated lossless transmission line having $N$ equal, but arbitrarily spaced, shunt admittances $Y$. The situation is depicted in figure 1, in which $Y_{0}$ is the characteristic admittance of the line, $\theta_{k}$ denotes the electrical length of the line section between the $k$ th and the $(k+1)$ th discontinuities, and $\Gamma_{k}$ represents the reflection coefficient looking toward the load at a point just to the left of the $k$ th discontinuity. Since input voltage standing-wave ratio $\rho_{\text {in }}$ and input reflection coefficient $\Gamma_{\text {in }}$ are definitely related by the equation

$$
\rho_{\text {in }}=\frac{1+\left|\Gamma_{\text {in }}\right|}{1-\left|\Gamma_{\text {in }}\right|}
$$

the problem of input VSWR minimization is equivalent to that of minimizing $\left|\Gamma_{\text {in }}\right|$.

\footnotetext{
${ }^{1}$ Presented at the URSI Spring Meeting, National Academy of Sciences, Washington, D.C., April 15-18, 1964.
} 
Figure 1. A transmission line with $\mathrm{N}$ equal discontinuities.

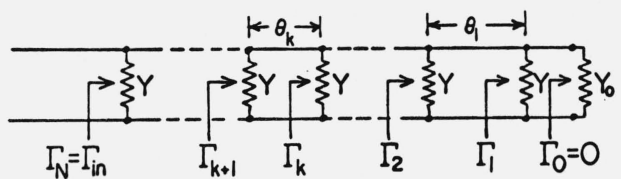

In terms of normalized admittances

$$
y_{k}=\frac{Y_{k}}{Y_{0}}
$$

where $Y_{k}$ is the admittance to the left of the $k$ th discontinuity looking toward the load, we can write

and

$$
\Gamma_{k}=\frac{1-y_{k}}{1+y_{k}}
$$

$$
\begin{aligned}
y_{k}=\frac{1-\Gamma_{k-1} e^{-2 j \theta_{k-1}}}{1+\Gamma_{k-1} e^{-2 j \theta_{k-1}}}+y, & \\
k & =1,2,3, \ldots, N .
\end{aligned}
$$

In our case, $y_{1}=y+1$,

$$
\begin{aligned}
& \Gamma_{1}=-\frac{y}{2+y}, \\
& \Gamma_{2}=\frac{\Gamma_{1}+\Gamma_{1} e^{-2 j \theta_{1}}+2 \Gamma_{1}^{2} e^{-2 j \theta_{1}}}{1-\Gamma_{1}^{2} e^{-2 j \theta_{1}}},
\end{aligned}
$$

and

$$
\Gamma_{k+1}=\frac{\Gamma_{1}+\Gamma_{k} e^{-2 j \theta_{k}}+2 \Gamma_{1} \Gamma_{k} e^{-2 j \theta_{k}}}{1-\Gamma_{1} \Gamma_{k} e^{-2 j \theta_{k}}} .
$$

It is convenient to normalize the reflection coefficients with respect to $\Gamma_{1}$ :

$$
\gamma_{k}=\frac{\Gamma_{k}}{\Gamma_{1}}
$$

and to define

$$
\beta_{k}=e^{-2 j \theta_{k}}
$$

We have $\gamma_{1}=1$ and, from (7),

$$
\gamma_{k+1}=\frac{\left(1+2 \Gamma_{1}\right) \beta_{k} \gamma_{k}+1}{-\Gamma_{1}^{2} \beta_{k} \gamma_{k}+1}
$$

If we write $u$ for $\gamma_{k+1}$ and $v$ for $\beta_{k} \gamma_{k}$, we see that (10) represents a bilinear transformation between the variables $u$ and $v$ with the following form: 


$$
u=\frac{a^{\prime} v+b^{\prime}}{c^{\prime} v+d^{\prime}}
$$

where

$$
\begin{aligned}
& a^{\prime}=1+2 \Gamma_{1} \\
& c^{\prime}=-\Gamma_{1}^{2} \\
& b^{\prime}=d^{\prime}=1 .
\end{aligned}
$$

It is the purpose of this paper to make use of some of the important properties of the bilinear transformation to minimize $\left|\gamma_{N}\right|$, and hence $\left|\Gamma_{N}\right|$ and $\rho_{N}\left(=\rho_{\text {in }}\right)$.

\section{Properties of Bilinear Transformation}

Some of the more important properties of a bilinear transformation will be reviewed here in preparation for their application to the problem at hand [Ford, 1929; Guillemin, 1951]. First of all, in order to have a one-to-one correspondence between all points in the $u$ - and $v$-planes, we require that

$$
a^{\prime} d^{\prime}-b^{\prime} c^{\prime}=\left(1+\Gamma_{1}\right)^{2} \neq 0,
$$

which is equivalent to demanding that $\Gamma_{1} \neq-1$. This requirement is always satisfied in practice since no short circuit is to be connected across the line. In order to achieve invariance of lengths in a certain neighborhood, we divide the $a^{\prime}, b^{\prime}, c^{\prime}, d^{\prime}$ coefficients by $\left(1+\Gamma_{1}\right)$ and rewrite (11) as

$$
u=\frac{a v+b}{c v+d}
$$

where

$$
\begin{gathered}
a=\frac{a^{\prime}}{1+\Gamma_{1}}=\frac{1+2 \Gamma_{1}}{1+\Gamma_{1}} \\
c=\frac{c^{\prime}}{1+\Gamma_{1}}=-\frac{\Gamma_{1}^{2}}{1+\Gamma_{1}} \\
b=d=\frac{b^{\prime}}{1+\Gamma_{1}}=\frac{d^{\prime}}{1+\Gamma_{1}}=\frac{1}{1+\Gamma_{1}} .
\end{gathered}
$$

Now we have the so-called normalization condition for the bilinear transformation:

$$
a d-b c=1 .
$$

Equation (16) can be rearranged to express $v$ as a function of $u$ as follows:

$$
v=\frac{d u-b}{-c u+a}
$$

Three invariant properties are of importance:

(i) Fixed points of transformation. These are points for which $u=v$ can be determined from 
either (16) or (21). We have

$$
u_{f 1}=v_{f 1}=\frac{1}{2 c}\left[(a-d)+\sqrt{(a+d)^{2}-4}\right]
$$

and

$$
u_{f 2}=v_{f 2}=\frac{1}{2 c}\left[(a-d)-\sqrt{(a+d)^{2}-4}\right]
$$

(ii) Invariance of the cross-ratio of four points. If we define the cross-ratio of four points, $u_{1}, u_{2}, u_{3}$, and $u_{4}$ in the $u$-plane as

$$
\left(u_{1} u_{2}, u_{3} u_{4}\right)=\frac{\left(u_{1}-u_{3}\right)\left(u_{2}-u_{4}\right)}{\left(u_{1}-u_{4}\right)\left(u_{2}-u_{3}\right)}
$$

then it is easy to prove by direct substitution that

$$
\left(u_{1} u_{2}, u_{3} u_{4}\right)=\left(v_{1} v_{2}, v_{3} v_{4}\right)
$$

(iii) Invariance of lengths-isometric circles. Isometric circles are the circles which represent the complete loci of points, in the neighborhood of which lengths and areas are unaltered in magnitude by a bilinear transformation. They are determined by setting $|d u / d v|=1$ and $|d v / d u|$ $=1$. Two relations are obtained:

and

$$
|c v+d|=1
$$

$$
|c u-a|=1 \text {. }
$$

Equation (26) represents the isometric circle of the direct transformation, $C_{d}$, having its center at

$$
O_{d}=-\frac{d}{c}=\frac{1}{\Gamma_{1}^{2}}
$$

and (27) specifies the isometric circle of the inverse transformation, $C_{i}$, with its center at

$$
O_{i}=\frac{a}{c}=-\frac{1}{\Gamma_{1}^{2}}\left(1+2 \Gamma_{1}\right)
$$

Both isometric circles have the same radius

$$
R=\frac{1}{|c|}=\left|\frac{1}{\Gamma_{1}^{2}}\left(1+\Gamma_{1}\right)\right|
$$

The invariance of the cross-ratio and the fact that the cross-ratio of four points on a circle is a positive real number establish the property that circles transform into circles. Further, the two fixed points of the present transformation are found to coalesce to one point, since $a+d=2$. Thus,

$$
u_{f}=v_{f}=-\frac{1}{\Gamma_{1}}
$$

This property signifies that the transformation is parabolic and that the isometric circles $C_{d}$ and $C_{i}$ are tangent to each other at the fixed point. 


\section{Two. Methods of Representation}

There are two methods for representing a bilinear transformation graphically. The conventional method is to rearrange (16) as

$$
u=\frac{a}{c}+\frac{\left(\frac{b}{c}-\frac{a d}{c^{2}}\right)}{v+\frac{d}{c}},
$$

which, in view of $(20)$, reduces to

$$
u=\frac{a}{c}-\frac{\frac{1}{c^{2}}}{v+\frac{d}{c}} .
$$

The bilinear transformation can then be interpreted as a combination of four operations in the complex plane:

(1) a translation, $v \rightarrow v+\frac{d}{c}$;

(2) an inversion, $v+\frac{d}{c} \rightarrow 1 /|c|^{2}\left(v+\frac{d}{c}\right)$;

(3) a rotation, $1 /|c|^{2}\left(v+\frac{d}{c}\right) \rightarrow-1 / c^{2}\left(v+\frac{d}{c}\right)$; and

(4) another translation, $-1 / c^{2}\left(v+\frac{d}{c}\right) \rightarrow \frac{a}{c}-1 / c^{2}\left(v+\frac{d}{c}\right)=u$.

A second, completely equivalent representation, known as the isometric-circle method [Ford, 1929; Bolinder, 1957], is possible for transforming a point $v$ to the corresponding point $u$ and vice versa. This method interprets the bilinear transformation as a combination of three steps as follows:

(1) an inversion with respect to the isometric circle $C_{d}$;

(2) a reflection with respect to the symmetry line of the two isometric circles $C_{d}$ and $C_{i}$; and

(3) a rotation around the center, $O_{i}$, of $C_{i}$ through an angle $-2 \arg (a+d)$.

A proof of the validity of the isometric-circle method of representation for the general case is contained in the appendix. The remainder of this paper will demonstrate the technique of applying the isometric-circle method to the problem of input VSWR minimization for a transmission line with multiple discontinuities.

\section{Minimization of Input VSWR for Two Equal Discontinuities}

We now consider the case for two equal discontinuities. Here $\Gamma_{\text {in }}=\Gamma_{2}$, and it is desired to minimize $\left|\Gamma_{2}\right|$ or $\left|\gamma_{2}\right|$. From (10), we have

$$
\gamma_{2}=\frac{\left(1+2 \Gamma_{1}\right) \beta_{1}+1}{-\Gamma_{1}^{2} \beta_{1}+1}
$$

which subsequently converts into the bilinear form of (16) with $u$ substituted for $\gamma_{2}$ and $v$ for $\beta_{1}\left(\gamma_{1}=1\right)$. 


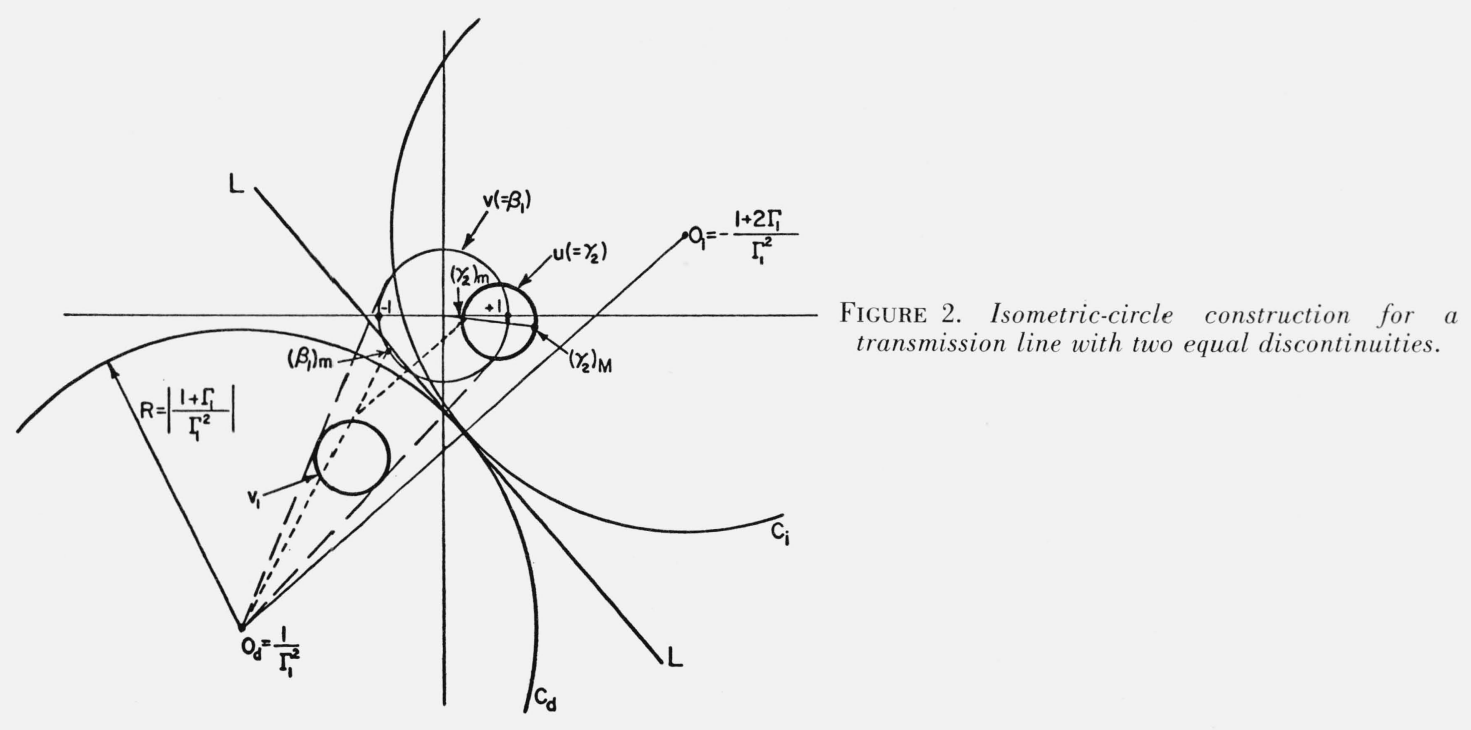

The isometric-circle method can be used directly to determine, for a given $\Gamma_{1}$ : (i) the minimum obtainable input VSWR, $\left(\rho_{\text {in }}\right)_{m}$; and (ii) the required electrical line length $\theta_{1}$ between the two discontinuities in order to achieve $\left(\rho_{\text {in }}\right)_{m}$.

Since the locus of $v=\beta_{1}=e^{-2 j \theta_{1}}$ for different values of $\theta_{1}$ is a unit circle centered at the origin, we proceed as follows (see fig. 2):

(1) Draw a circle of unit radius (locus of $v$ ) at the origin.

(2) Draw the two isometric circles $C_{d}$ and $C_{i}$ with equal radius $R=1 /|c|=\left|\left(1+\Gamma_{1}\right) / \Gamma_{1}^{2}\right|$ at centers $O_{d}=1 / \Gamma_{1}^{2}$ and $O_{i}=-\left(1+2 \Gamma_{1}\right) / \Gamma_{1}^{2}$ respectively, and draw the symmetry line (common tangent) $L-L$.

(3) Invert the unit circle with respect to $C_{d}$ to get the $v_{1}$ circle.

(4) Reflect the $v_{1}$-circle about line $L-L$ to get the locus of $u\left(=\gamma_{2}=\Gamma_{2} / \Gamma_{1}\right)$.

(5) From figure 2 it is a simple matter to determine the minimum magnitude of $\gamma_{2},\left|\gamma_{2}\right|_{m}$. From $\left|\gamma_{2}\right|_{m},\left|\Gamma_{2}\right|_{m}=\left|\Gamma_{1}\right|\left|\gamma_{2}\right|_{m}$, and $\left(\rho_{\text {in }}\right)_{m}$ can be found:

$$
\left(\rho_{\mathrm{in}}\right)_{m}=\frac{1+\left|\Gamma_{2}\right|_{m}}{1-\left|\Gamma_{2}\right|_{m}}
$$

(6) The required line length $\left(\theta_{1}\right)_{m}$ can be obtained from (33) by substituting $\left(\gamma_{2}\right)_{m}$ for $\gamma_{2}$ :

$$
\left(\beta_{1}\right)_{m}=e^{-2 j\left(\theta_{1}\right)_{m}}=\frac{\left(\gamma_{2}\right)_{m}-1}{1+2 \Gamma_{1}+\Gamma_{1}^{2}\left(\gamma_{2}\right)_{m}} .
$$

Since $a+d=2$ in our case, no rotation is needed ( $\phi=0$ in (53) and in fig. 6) after reflection about the symmetry line $L-L$, and the point $\left(\beta_{1}\right)_{m}$ on the $v$-circle can be very simply determined graphically as shown. With $\left(\beta_{1}\right)_{m}$ and $\left(\theta_{1}\right)_{m}$, the solution of the problem is complete.

The question may be asked whether it is possible to get a perfect match $\left(\gamma_{2}=0\right.$ or $\left.\rho_{\text {in }}=1\right)$ with two equal discontinuities. From (33) we see that this occurs only when

$$
\beta_{1}=e^{-2 j \theta_{1}}=-\frac{1}{1+2 \Gamma_{1}},
$$

which requires

$$
\left|\frac{1}{1+2 \Gamma_{1}}\right|=1 \text {. }
$$


Equation (37) limits the values of $\Gamma_{1}$ for perfect match to lie on a circle of radius $1 / 2$ centered at $-1 / 2$ in the complex plane. Hence, we are able to predict, for example, that a perfect match is unattainable for two equal discontinuities with $\Gamma_{1}=-1 / 2+j 3 / 4$; but, if $\Gamma_{1}=-1 / 2+j 1 / 2$, a perfect match can be obtained and the required $\theta_{1}$ readily determined from (36).

\section{Three or More Equal Discontinuities}

When there are three equal discontinuities, it is necessary to analyze not only (33) but also

$$
\gamma_{3}=\frac{\left(1+2 \Gamma_{1}\right) \beta_{2} \gamma_{2}+1}{-\Gamma_{1}^{2} \beta_{2} \gamma_{2}+1}
$$

and input VSWR minimization can be effected by repeated application of the bilinear transformation. In order to avoid confusion and clutter, it is advantageous to consider each transformation as a mapping from one plane onto another. Thus, points on the unit circle in the $\beta_{1}$-plane are first mapped into a circle in the $\gamma_{2}$-plane, and points on the $\beta_{2} \gamma_{2}$-circle, centered at the origin of the $\gamma_{2}$-plane, are then mapped into another circle in the $\gamma_{3}$-plane.

The transformation of the unit circle in the $\beta_{1}$-plane into a $\gamma_{2}$-circle has been illustrated in figure 2 for the two-discontinuity case. The product $\beta_{2} \gamma_{2}$ will be a family of concentric circles at the origin in the $\gamma_{2}$-plane as shown in figure 3(a), the radii of the circles being dependent upon the value of $\left|\gamma_{2}\right|$. A second application of the isometric-circle method of bilinear transformation will transform the family of concentric $\beta_{2} \gamma_{2}$-circles into a family of $\gamma_{3}$-circles as shown in either figure $3(\mathrm{~b})$ or figure 3(c). From (10), we note that the origin of the $\gamma_{2}$-plane transforms into the $(1, j 0)$ point of the $\gamma_{3}$-plane. Because of the conformal nature of the transformation, the transformed family of $\gamma_{3}$-circles will not intersect and will be nested about the $(1, j 0)$ point. The smallest circle $\beta_{2}\left(\gamma_{2}\right)_{m}$ in the $\gamma_{2}$-plane transforms to the smallest circle in the $\gamma_{3}$-plane and the largest circle $\beta_{2}\left(\gamma_{2}\right)_{M}$ transforms to the largest.

The situation in figure 3(b) indicates that a perfect match is not possible because the largest of the $\gamma_{3}$-circles does not encompass or pass through the origin. The value of $\left|\gamma_{3}\right|_{m}$, which yields the minimum $\left|\Gamma_{3}\right|_{m}$ or $\left(\rho_{\text {in }}\right)_{m}$, can be readily determined from the figure. It is noted that, in order to minimize $\left|\gamma_{3}\right|$, it is necessary to make $\left|\gamma_{2}\right|$ maximum. With $\left(\gamma_{3}\right)_{m}$ and $\left(\gamma_{2}\right)_{M}$ determined, inverse transformations will give the required electrical line lengths $\theta_{1}$ and $\theta_{2}$ between discontinuities.

Figure 3(c) shows a case where a perfect match is possible. The largest $\gamma_{3}$-circle now encloses the origin; hence one member of the family of $\gamma_{3}$-circles does pass through it, satisfying the condition for a perfect match.

In general, it would be desirable if it could be predicted, by a simple computation without the elaborate transformation procedure, whether a perfect match is attainable with three discontinuities for a given $\Gamma_{1}$. As will be shown in a later section, the range of values of $\Gamma_{1}$, for which a perfect match can be made, can be determined without difficulty when $\Gamma_{1}$ is real; but, when $\Gamma_{1}$ is complex, the analytical relation between $\left|\gamma_{2}\right|_{M}$ and $\left|\Gamma_{1}\right|$ is too complicated to be of use.

When there are more than three discontinuities, the same technique can be applied. Here, a family of circles at the origin extending from $\beta_{k}\left(\gamma_{k}\right)_{m}$ to $\beta_{k}\left(\gamma_{k}\right)_{M}$ in the $\gamma_{k}$-plane transforms to a

FiguRE 3. VSWR minimization for line with three equal discontinuities:

(a) $\beta_{2} \gamma_{2}$-circles in $\gamma_{2}$-plane

(b) $\gamma_{3}$-circles when a perfect match is not possible

(c) $\gamma_{3}$-circle when a perfect match is possible.

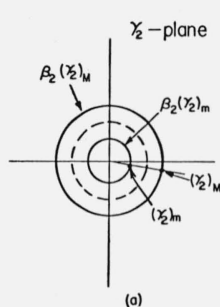

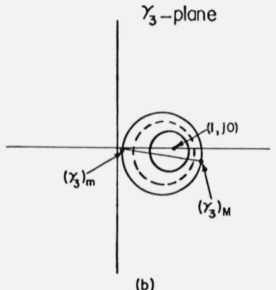

(b)

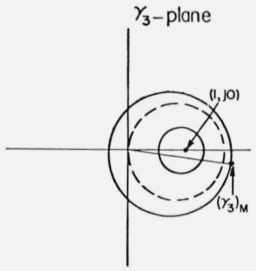

(c) 
family of nested circles enclosing the $(1, j 0)$ point in the $\gamma_{k+1}$-plane. If the magnitude relation

$$
\left|\gamma_{N-1}\right|=\frac{1}{\left|1+2 \Gamma_{1}\right|}
$$

can be satisfied, then it is possible to make $\gamma_{N}=0$, and a perfect match can be achieved with $N$ discontinuities. If a perfect match is not attainable, then $\left|\gamma_{N}\right|_{m}$ determines $\left(\rho_{\text {in }}\right)_{m}$, and the required electrical line lengths $\theta_{N-1}, \theta_{N-2}, \ldots, \theta_{2}$ and $\theta_{1}$ can be found from $\left(\gamma_{N}\right)_{m},\left(\gamma_{N-1}\right)_{M}, \ldots,\left(\gamma_{3}\right)_{M}$, and $\left(\gamma_{2}\right)_{M}$, respectively, by successive inverse transformations.

Example 1. Consider the case of three equal discontinuities:

$$
y_{1}=y_{2}=y_{3}=\frac{Y_{1}}{Y_{0}}=0.674 / 77^{\circ} \text {. }
$$

It is desired to determine the electrical lengths $\theta_{1}$ and $\theta_{2}$ required to effect a perfect match.

From (5) we compute

$$
\Gamma_{1}=0.300 \angle 240^{\circ}=-0.150-j 0.260
$$

and

$$
1+2 \Gamma_{1}=0.700-j 0.520=0.872\left\lfloor-36.6^{\circ} .\right.
$$

For a perfect match, we require

$$
\left|\gamma_{2}\right|=\left|\frac{1}{1+2 \Gamma_{1}}\right|=\frac{1}{0.872}=1.15 \text {. }
$$

The unit circle in the $\beta_{1}$-plane is mapped into a $\gamma_{2}$-circle as shown in figure 4 , either by converting three points or by isometric-circle construction. Two points exist on the circle at which $\left|\gamma_{2}\right|=1.15$; hence a perfect match can be obtained with three discontinuities in two ways. We have

$$
\gamma_{2}=1.15 e^{j 42.6^{\circ}} \text {, or } \gamma_{2}=1.15 e^{-j 45.8^{\circ}} \text {. }
$$

In order to make

$$
\beta_{2} \gamma_{2}=-\frac{1}{1+2 \Gamma_{1}}
$$

or

$$
e^{-2 j \theta_{2}} \gamma_{2}=1.15 e^{j\left(36.6^{\circ}-180^{\circ}\right)},
$$

we determine two values of $\theta_{2}$ by the following relation:

$$
\theta_{2}=\frac{1}{2}\left(180^{\circ}-36.6^{\circ}+\arg \gamma_{2}\right)
$$

from which we find $\theta_{2}=93.0^{\circ}$ or $48.8^{\circ}$. Substitution of $k=1$ and rearrangement of (10) gives

$$
\beta_{1}=e^{-2 j \theta_{1}}=\frac{\gamma_{2}-1}{\left(1+2 \Gamma_{1}\right)+\Gamma_{1}^{2} \gamma_{2}}
$$

from which we obtain $\theta_{1}=114.6^{\circ}$ or $34.4^{\circ}$.

With these values of $\theta_{1}$ and $\theta_{2}$ we can effect a perfect match at the input $\left(\rho_{\text {in }}=1\right)$. As a result of this solution, the problem of $3 n(n=1,2$, . .) discontinuities has also been solved. 


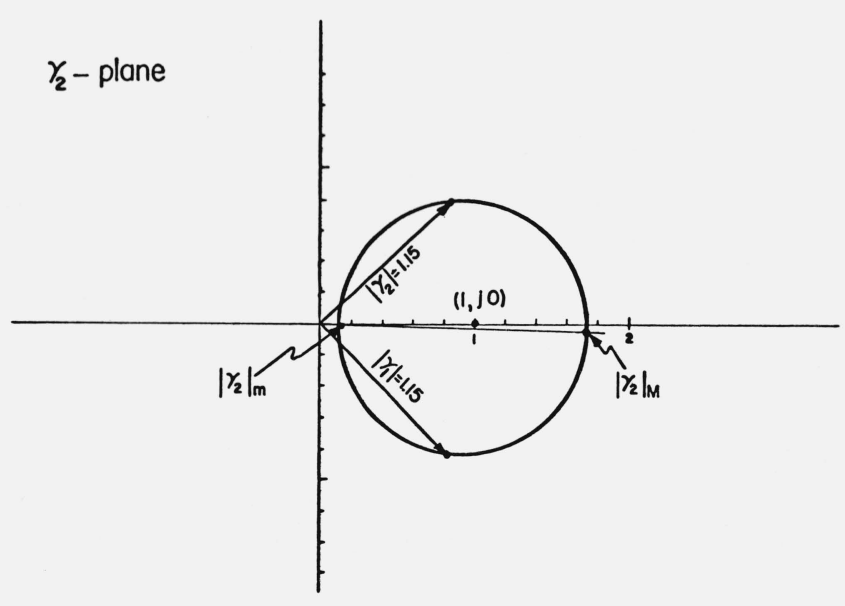

\section{N Equal, Real Discontinuities}

We consider now the general case of $N$ equal, real discontinuities. Because of the way in which $y_{k}$ and $\Gamma_{k}$ are defined, $y_{1}$ will be real and positive and $\Gamma_{1}$ will be real and negative. Both isometric circles $C_{d}$ and $C_{i}$ are now symmetric about the real axis; the point $\left(\beta_{k} \gamma_{k}\right)_{M}=\left(\gamma_{k}\right)_{M}$ transforms into $\left(\gamma_{k+1}\right)_{M}$, and the point $\left(\beta_{k} \gamma_{k}\right)_{m}=-\left(\gamma_{k}\right)_{M}$ transforms into $\left(\gamma_{k+1}\right)_{m}$. With these specializations, it is now possible to tabulate $\left(\gamma_{k}\right)_{M}$ and $\left(\gamma_{k}\right)_{m}$ values for a general $\Gamma_{1}$ (see table 1 ).

TABLE 1.

\begin{tabular}{c|c|c|c|c}
\hline \hline$k$ & $\left(\beta_{k} \gamma_{k}\right)_{M}=\left(\gamma_{k}\right)_{M}$ & $\left(\gamma_{k+1}\right)_{M}$ & $\left(\beta_{k} \gamma_{k}\right)_{m}=-\left(\gamma_{k}\right)_{M}$ & $\left(\gamma_{k+1}\right)_{m}$ \\
\hline 1 & 1 & $\frac{2}{1-\Gamma_{1}}$ & -1 & $-\frac{2 \Gamma_{1}}{1+\Gamma_{1}^{2}}$ \\
\hline 2 & $\frac{2}{1-\Gamma_{1}}$ & $\frac{3}{1-2 \Gamma_{1}}$ & $-\frac{2}{1-\Gamma_{1}}$ & $-\frac{\left(1+5 \Gamma_{1}\right)}{1-\Gamma_{1}+2 \Gamma_{1}^{2}}$ \\
\hline$\vdots$ & $\vdots$ & $\vdots$ & $\vdots$ & $\vdots$ \\
\hline$N-2$ & $\frac{N-2}{1-(N-3) \Gamma_{1}}$ & $\frac{j-1}{1-(N-2) \Gamma_{1}}$ & & \\
\hline$N-1$ & & & $-\frac{N-1}{1-(N-2) \Gamma_{1}}$ & $-\frac{(N-2)+(3 N-4) \Gamma_{1}}{1-(N-2) \Gamma_{1}+(N-1) \Gamma_{1}^{2}}$ \\
\hline
\end{tabular}

Table 1 contains all the information necessary to evaluate $\left(\rho_{\text {in }}\right)_{m}$ for the case of $N$ equal, real discontinuities. When a perfect match is possible, $\left(\gamma_{k}\right)_{m}$ becomes negative or zero for a certain $k$. From the table we observe that a perfect match is not possible with two equal and real discontinuities, since $\left(\gamma_{2}\right)_{m}$ is always positive for negative $\Gamma_{1}$. With three discontinuities, however, a perfect match could be achieved if the value of $\Gamma_{1}$ lies within the range: $-1 / 5 \leqslant \Gamma_{1} \leqslant 0$. In general, for $N$ real and equal discontinuities, a perfect match is possible if

$$
-\left(\frac{N-2}{3 N-4}\right) \leqslant \Gamma_{1} \leqslant 0
$$

which is the same as requiring

$$
0 \leqslant Y \leqslant\left(\frac{N-2}{N-1}\right) Y_{0}
$$


as $N \longrightarrow \infty,(40)$ and (41) become approximately

$$
-\frac{1}{3} \leqslant \Gamma_{1} \leqslant 0
$$

and

$$
0 \leqslant Y \leqslant Y_{0}
$$

When a perfect match is not possible, the minimum obtainable input VSWR after $N$ discontinuities will be

$$
\left(\rho_{\text {in }}\right)_{m}=\frac{1+\left|\Gamma_{1}\left(\gamma_{N}\right)_{m}\right|}{1-\left|\Gamma_{1}\left(\gamma_{N}\right)_{m}\right|}=\frac{1+(4 N-5) \Gamma_{1}^{2}}{1-2(N-2) \Gamma_{1}-(2 N-3) \Gamma_{1}^{2}} .
$$

The electrical line lengths between the discontinuities should be $\theta_{k}=\pi$ for $k=1,2, \ldots ., N-2$, and $\theta_{N-1}=\pi / 2$. All discontinuities except the one nearest to the input are effectively put in parallel which are then reflected through a quarter-wavelength section and added to $Y$. Since $Y$ would already be too large to satisfy (41) for a perfect match, this arrangement would reflect a minimum additional conductance at $Y_{N}$, hence yielding a minimum input VSWR.

\section{Input VSWR Minimization for Unequal Discontinuities}

When the discontinuities across a line are unequal, VSWR minimization depends upon the order in which they appear. Referring to figure 5 , if we call the first discontinuity $Y$, the second $\alpha_{2} Y, \ldots$, and the $k$ th $\alpha_{k} Y$, where the $\alpha_{k}$ 's may be complex $\left(\alpha_{1}=1\right)$, we may write, from (4),

$$
y_{k}=\frac{1-\Gamma_{k-1} e^{-2 j \theta_{k-1}}}{1+\Gamma_{k-1} e^{-2 j \theta_{k-1}}}+\alpha_{k} y .
$$

Also,

$$
\gamma_{2}=\frac{\Gamma_{2}}{\Gamma_{1}}=\frac{\alpha_{2}+\left(1+\Gamma_{1}+\alpha_{2} \Gamma_{1}\right) \beta_{1}}{\left(1+\Gamma_{1}-\alpha_{2} \Gamma_{1}\right)-\alpha_{2} \Gamma_{1}^{2} \beta_{1}}
$$

and, in general,

$$
\gamma_{k+1}=\frac{\Gamma_{k+1}}{\Gamma_{1}}=\frac{\alpha_{k+1}+\left(1+\Gamma_{1}+\alpha_{k+1} \Gamma_{1}\right) \beta_{k} \gamma_{k}}{\left(1+\Gamma_{1}-\alpha_{k+1} \Gamma_{1}\right)-\alpha_{k+1} \Gamma_{1}^{2} \beta_{k} \gamma_{k}}
$$

It is obvious that (46) represents a bilinear transformation between the two variables $\gamma_{k+1}$ and $\left(\beta_{k} \gamma_{k}\right)$ and can be put in the form of (11). Furthermore, we find that $a^{\prime} d^{\prime}-b^{\prime} c^{\prime}=\left(1+\Gamma_{1}\right)^{2}$, which is exactly the same as (15) for the equal-discontinuity case. Hence, input VSWR minimization for unequal discontinuities can follow the same techniques which have been developed previously for equal discontinuities. Because of the changing $a^{\prime}, b^{\prime}, c^{\prime}, d^{\prime}$ values, however, no prediction

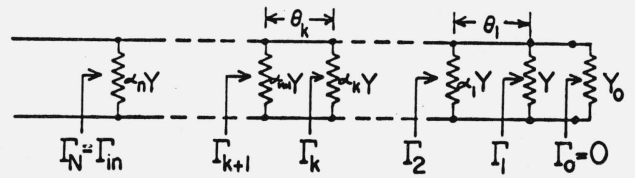

Figure 5. A transmission line with unequal discontinuities. 
on perfect match conditions is possible. The procedure would in general be more involved, as the nesting point would now change its location on each application of the bilinear transformation.

Example 2. Consider the case of three real, unequal discontinuities;

$$
y_{1}=2, \quad y_{2}=3, \quad \text { and } y_{3}=5,
$$

which means that $\alpha_{2}=3 / 2$ and $\alpha_{3}=5 / 2$. It is desired to minimize $\left|\Gamma_{3}\right|$ or $\rho_{\text {in }}$.

From (5), we compute $\Gamma_{1}=-1 / 2$. Substitution of the values of $\alpha_{2}, \alpha_{3}$, and $\Gamma_{1}$ in (44) and (45) results in two equations:

$$
\gamma_{2}=\frac{12-2 \beta_{1}}{10-3 \beta_{1}}
$$

and

$$
\gamma_{3}=\frac{20-6 \beta_{2} \gamma_{2}}{14-5 \beta_{2} \gamma_{2}}
$$

Mapping of the points $\beta_{1}=+1$ and $\beta_{1}=-1$ yields the points $\left(\gamma_{2}\right)_{M}=10 / 7$ and $\left(\gamma_{2}\right)_{m}=14 / 13$ respectively. A second mapping of the $\beta_{2} \gamma_{2}$-circle into a $\gamma_{3}$-circle transforms the point $\left(\beta_{2} \gamma_{2}\right)_{m}=-\left(\gamma_{2}\right)_{M}$ into the point for $\left(\gamma_{3}\right)_{m}=50 / 37$. Thus, we have

$$
\left|\Gamma_{3}\right|_{m}=\left|\Gamma_{1}\left(\gamma_{3}\right)_{m}\right|=25 / 37
$$

and

$$
\left(\rho_{\text {in }}\right)_{m}=\frac{1+\left|\Gamma_{3}\right|_{m}}{1-\left|\Gamma_{3}\right|_{m}}=5 \frac{1}{6}
$$

for $\theta_{1}=\pi$ and $\theta_{2}=\pi / 2$. The minimum obtainable input VSWR will be different if the order of $y_{1}, y_{2}$, and $y_{3}$ is changed. For instance, if, instead of in the $2-3-5$ order, the three discontinuities are connected in the $5-3-2$ order, we find $\left(\rho_{\text {in }}\right)_{m}=2 \frac{1}{3}$.

\section{Conclusion}

We have shown that input VSWR minimization for a transmission line with multiple discontinuities joined by line sections can be achieved by using the isometric-circle method for interpreting a bilinear transformation. The basic technique can be applied in a repeated fashion to the general case of a line with $N$ unequal, complex-admittance discontinuities. It enables us to determine not only the minimum attainable input VSWR but also the required line lengths between the successive discontinuities. If the $N$ discontinuities are real and equal, it is possible to predict the range of the reflection coefficient $\Gamma_{1}$ of the individual discontinuities for which a perfect match would be obtainable. A general expression for the minimum input VSWR has also been found when $\Gamma_{1}$ falls outside of the specified range, making a perfect match impossible to obtain.

\section{Appendix. Isometric-Circle Method of Representing Bilinear Transformation}

Let us rewrite the bilinear transformation in (32) as

$$
u-\frac{a}{c}=-\frac{1}{c^{2}\left(v+\frac{d}{c}\right)} .
$$


FIGURE 6. Isometric representation of bilinear transformation.

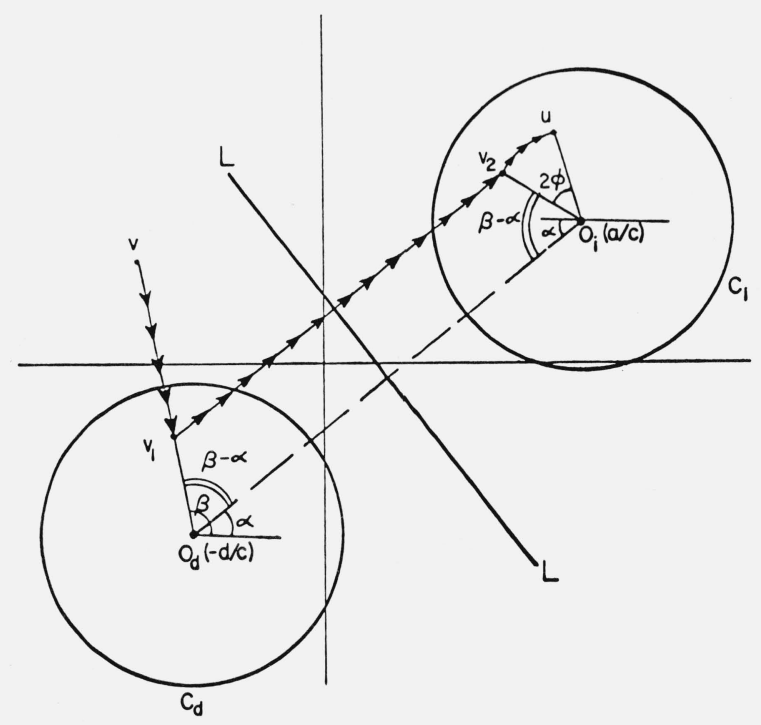

Two relations are implied by (47): a magnitude relation and an angle relation. The magnitude relation is

$$
\left|u-\frac{a}{c}\right|=\frac{1}{|c|^{2}\left|v+\frac{d}{c}\right|} .
$$

Noting from (28) and (29) that $-d / c$ and $a / c$ are respectively the locations of the centers $O_{d}$ and $O_{i}$ of the isometric circles $C_{d}$ and $C_{i}$, we see from (48) that the distance between $O_{i}$ and $u$ should be equal to the distance between $O_{d}$ and the point obtained by inverting $v$ with respect to $C_{d}$ which has a radius $R=1 /|c|$. This magnitude relation is satisfied by the construction in figure 6 :

$$
\overline{O_{d} v_{1}}=\overline{O_{i} v_{2}}=\overline{O_{i} u}
$$

In figure $6, C_{d}$ and $C_{i}$ are the two isometric circles, $L-L$ is the symmetry line, $v_{1}$ is the inversion point of $v$ with respect to $C_{d}$, and $v_{2}$ is the reflection point of $v_{1}$ about $L-L$.

That the angle relation implied in (47),

$$
\begin{aligned}
\arg \left(u-\frac{a}{c}\right) & =\arg \left[-\frac{1}{c^{2}\left(v+\frac{d}{c}\right)}\right] \\
& =\pi+\arg \left[\frac{1}{c^{2}\left(v+\frac{d}{c}\right)}\right],
\end{aligned}
$$

is satisfied by the construction in figure 6 can be more easily seen by rewriting (50) in the following form:

$$
\begin{aligned}
\arg \left(u-\frac{a}{c}\right) & =\pi+\arg \left[\left(\frac{a+d}{c}\right)^{2} \frac{1}{\left(v+\frac{d}{c}\right)} \frac{1}{(a+d)^{2}}\right] \\
& =\pi+(2 \alpha-\beta-2 \phi),
\end{aligned}
$$


where

$$
\begin{aligned}
& \alpha=\arg \left(\frac{a+d}{c}\right) \\
& \beta=\arg \left(v+\frac{d}{c}\right) \\
& \phi=\arg (a+d) .
\end{aligned}
$$

In figure 6 , the angle which the line $O_{i} u$ makes with the horizontal is seen to be $\pi-(\beta-\alpha-\alpha)-2 \phi$, which is the same as the right-hand side of (51).

The above has demonstrated that a point $v$ can be transformed to the point $u$ in accordance with (47) by (1) inverting $v$ in isometric circle $C_{d}\left(v \rightarrow v_{1}\right)$, (2) reflecting $v_{1}$ with respect to symmetry line $L-L\left(v_{1} \rightarrow v_{2}\right)$, and (3) rotating $v_{2}$ about $O_{i}$ in a clockwise direction by angle $2 \phi\left(v_{2} \rightarrow u\right)$, as shown in figure 6.

\section{References}

Bolinder, E. F. (June 14, 1957), Impedance and power transformations by the isometric circle method and non-Euclidean hyperbolic geometry, MIT Res. Lab. of Electronics, Tech. Report No. 312.

Ford, L. R. (1929), Automorphic Functions, ch. 1 (McGraw-Hill Book Co., New York, N.Y.).

Guillemin, E. A. (1951), The Mathematics of Circuit Analysis, ch. VI, Art. 24 (John Wiley \& Sons, Inc., New York, N.Y.).

(Paper 69D9-562) 